\title{
Vaccine Design and Animal Testing
}

National Cancer Institute

\section{Source}

National Cancer Institute. Vaccine Design and Animal Testing. NCI Thesaurus. Code C15865.

Apply findings from basic, epidemiologic, and clinical research to the design and evaluation of vaccine strategies in laboratory studies and animal models, and foster collaboration with industry in the research and development of candidate vaccines. 